Article

\title{
Approximate Controllability of Fully Nonlocal Stochastic Delay Control Problems Driven by Hybrid Noises
}

\author{
Lixu Yan ${ }^{*}+$ and Yongqiang Fu ${ }^{\dagger}$ \\ School of Mathematics, Harbin Institute of Technology, Harbin 150001, China; fuyongqiang@hit.edu.cn \\ * Correspondence: yanlxmath@163.com \\ † These authors contributed equally to this work.
}

check for updates

Citation: Yan, L.X.; Fu, Y.

Approximate Controllability of Fully Nonlocal Stochastic Delay Control Problems Driven by Hybrid Noises. Fractal Fract. 2021, 5, 30.

https://doi.org/10.3390/

fractalfract5020030

Academic Editor: Mirko D'Ovidio

Received: 10 March 2021

Accepted: 8 April 2021

Published: 12 April 2021

Publisher's Note: MDPI stays neutral with regard to jurisdictional claims in published maps and institutional affiliations.

Copyright: (c) 2021 by the authors. Licensee MDPI, Basel, Switzerland. This article is an open access article distributed under the terms and conditions of the Creative Commons Attribution (CC BY) license (https:// creativecommons.org/licenses/by/ $4.0 /)$.

\begin{abstract}
In this paper, a class of time-space fractional stochastic delay control problems with fractional noises and Poisson jumps in a bounded domain is considered. The proper function spaces and assumptions are proposed to discuss the existence of mild solutions. In particular, approximate strategy is used to obtain the existence of mild solutions for the problem with linear fractional noises; fixed point theorem is used to achieve the existence of mild solutions for the problem with nonlinear fractional noises. Finally, the approximate controllability of the problems with linear and nonlinear fractional noises is proved by the property of mild solutions.
\end{abstract}

Keywords: fully nonlocal derivative operator; mild solution; approximate controllability; fractional Brownian motion; Poisson jump

MSC: 60H15; 35A01; 47H06

\section{Introduction}

We study a class of fully nonlocal stochastic control problems with delay in a bounded domain $\mathcal{O} \subset \mathbb{R}^{N}$ :

$$
\left\{\begin{array}{l}
{ }_{0}^{C} D_{t}^{\alpha} y(t, x)+(-\Delta)^{\gamma} y(t, x)=f\left(t, x, y_{\tau}(t, x)\right)+[\mathcal{B} v(t)](x) \\
\quad+g\left(t, y_{\tau}(t, x)\right) \frac{d B^{H}(t)}{d t}+\int_{U} h\left(t, x, y_{\tau}(t, x) ; u\right) \tilde{\theta}(d u), t \in(0, T], x \in \mathcal{O}, \\
y(t, x)=\varphi(t, x), \text { a.s., } t \in[-\tau, 0], x \in \mathcal{O}, \\
y(t, x)=0, \text { a.s., } t \in[-\tau, T], x \in \mathbb{R}^{N} \backslash \mathcal{O},
\end{array}\right.
$$

where $\alpha \in(1 / 2,1), \gamma \in(1 / 2,1), T \in(0, \infty)$, and $N>2 \gamma .{ }_{0}^{C} D_{t}^{\alpha}$ is the time Caputo fractional derivative and $(-\Delta)^{\gamma}$ is the fractional Laplacian. Constant $\tau>0$ is a fixed time delay. Notation $y_{\tau}$ denotes the history of $y$ with respect to time, i.e., $y_{\tau}(t, x)=y(t-\tau, x)$ for $t \in[0, T]$ and $x \in \mathcal{O}$. Function $f(t, x, y):[0, T] \times \mathcal{O} \times C_{\mathcal{F}_{t}} \rightarrow \mathbb{R}$ denotes the drift term. $g \in \mathscr{L}_{2}^{0}\left(W, L^{2}(\mathcal{O})\right)$ and $B^{H}$ is a $W$-valued cylindrical fractional Brownian motion with Hurst index $H \in(1 / 2,1)$, where $W$ is a given separable Hilbert space. Let $V$ be a Hilbert space. Control $v \in L^{2}([0, T], V)$ and operator $\mathcal{B} \in \mathscr{L}\left(V, L^{2}(\mathcal{O})\right)$. Let $(U, \mathscr{B}(U), e)$ be a $\sigma$-finite measurable space. Function $h(t, x, y ; u):[0, T] \times \mathcal{O} \times C_{\mathcal{F}_{t}} \times U \rightarrow \mathbb{R}$ and $\tilde{\theta}$ denotes the compensated Poisson martingale measure. We illustrate each term of the problem (1) in Section 2.

Time fractional derivatives are used to describe phenomenon of early arrival or to tail in time. Spatial fractional derivatives are more suitable for describing nonlocal and scale effects. There are many applications of fractional derivatives in anomalous diffusion, random walk, nonlocal elasticity, and memory materials, see [1-7] and the references therein. The fractional derivatives in problem (1) are constant-order fractional derivatives. In recent years, there has been a series of papers studying variable-order fractional derivatives and distributed-order fractional derivatives. These general fractional 
derivatives are applied to model the complex interaction and superposition systems of nonlocal effects and memory effects on multi-scales, see [8-11]. in $\mathbb{R}^{N}$ :

When $\alpha=1, \gamma \in(0,1), H=1$, and $h \equiv 0$, Wang [12] discussed the following equation

$$
d y(t, x)+(-\Delta)^{\gamma} y(t, x)=f\left(t, x, y_{\tau}(t, x)\right)+g\left(t, y_{\tau}(t, x)\right) d B(t),
$$

where $B$ is a Wiener process. He obtained the existence and uniqueness of solutions and weak pullback mean random attractors. Mohammed [13] proved the existence of approximate solutions on a bounded domain to the time-fractional case, i.e., $\alpha \in(1 / 2,1)$, $\gamma=1, H=1$ and $h \equiv 0$. However, we consider the time-space fractional case, which is called the fully nonlocal case.

In the last decade, fully nonlocal partial differential equations have attracted great attention. Several different approaches to simulate fully nonlocal PDE were presented in [14-18]. Fundamental solutions for fully nonlocal PDE problems were discussed in [19-21]. These results encourage researchers to study the fractional stochastic partial differential equations (f-SPDE). For example, f-SPDE driven by fractional Brownian motions with delay were considered in [22-24], and the existence of mild solutions was obtained under suitable assumptions. Caraballo, Garrido-Atienza and Taniguchi [25] considered the following problem driven by linear fractional noise:

$$
\left\{\begin{array}{l}
d y(t)=A y(t) d t+f\left(t, y_{\tau}(t)\right) d t+g(t) d B^{H}(t), t \in(0, T] \\
y(t)=y_{0}(t) \in X, t \in[-\tau, 0]
\end{array}\right.
$$

where $A$ is a bounded abstract operator and $X$ is a Hilbert space. They obtained the existence and uniqueness of mild solutions. Li [23] discussed problem (2) with fractional time derivative, i.e., ${ }_{0}^{C} D_{t}^{\alpha} y(t)$ instead of $d y(t)$ in (2) and established the existence and uniqueness of mild solution by approximate method when indexes $s \in(1 / 2,1]$ and $H \in(1 / 2,1)$. As for nonlinear fractional noises, Pei and $\mathrm{Xu}$ [24] considered the following problem:

$$
\left\{\begin{array}{l}
d y(t)=A(t, y(t)) d t+f\left(t, y_{\tau}(t)\right) d t+g\left(t, y_{\tau}(t)\right) d B(t)+\int_{U} h\left(t, y_{\tau}(t) ; u\right) \tilde{\theta}(d u, d t), t \in(0, T] \\
y(t)=\varphi(t) \in C_{\mathcal{F}_{0}}([-\tau, 0] ; X), t \in[-\tau, 0] .
\end{array}\right.
$$

The existence and uniqueness of mild solution were obtained under Lipschitz-type conditions. For more papers, we recommend [26,27].

We are interested in the controllability of problem (1). Controllability is a basic topic in control theory. It has wild applications in the real world, see $[28,29]$ and the references therein. Recently, approximate controllability of f-SPDE was studied by many researchers. In [30], Lakhel discussed a class of integro-differential equations with linear fractional noises,

$$
\begin{aligned}
d[y(t)+G(t, y(t-r(t)))]= & {[A y(t)+G(t, y(t-r(t)))+H v(t)] d t } \\
& +\int_{0}^{t} B(t-s)[y(s)+G(s, y(s-r(s)))] d s d t \\
& +F(t, y(s-r(s))) d t+Q(t) d B^{H}(t), t \in[0, T] .
\end{aligned}
$$

By means of resolvent operators, controllability of (3) was obtained when Hurst index $H \in(1 / 2,1)$. In [31], Ahmed considered the following equation driven by nonlinear fractional noises and Poisson jumps,

$$
\begin{aligned}
{ }_{0}^{C} D_{t}^{\alpha}[y(t)-G(t, y(t))]= & A[y(t)-G(t, y(t))]+\mathcal{B} v(t)+\int_{0}^{t} A_{1}(t-s)[y(s)-G(s, y(s))] d s \\
& +f(t, y(t))+g(t, y(t)) \frac{d B^{H}(t)}{d t}+\int_{U} h(t, y(t) ; u) \tilde{\theta}(d u), t \in[0, T],
\end{aligned}
$$


where $\alpha \in(1,2)$ and $H \in(1 / 2,1)$. Ahmed proved the approximate controllability under a uniform boundness condition

$$
\|f(t, y(t))\|_{X}+\|g(t, y(t))\|_{\mathscr{L}_{2}^{0}}+\int_{U}\|h(t, y(t) ; u)\|_{X} e(d u) \leq M .
$$

For more papers, we recommend [32,33]. Note that (4) is a common condition to discuss the approximate controllability of control problems, see, for examples, refs [34-36]. However, in this paper, we obtain the approximate controllability by the property of mild solutions.

Problem (1) strongly depends on the ranges of $\alpha, \gamma, H$, and $N$. In this paper, we suppose $1 / 2<\alpha, \gamma, H<1$, and $N>2 \gamma$. Compared with the aforementioned papers, there are two main contributions in this paper:

1. Utilize a reasonable framework of mild solutions and overcome the complex calculations caused by not only fractional differential operators but also fractional Brownian motions and Poisson jumps. Establish the existence and uniqueness of the mild solution to fully nonlocal stochastic delay control problems with both linear and nonlinear fractional noise by two different methods.

2. Establish sufficient conditions to the approximate controllability of fully nonlocal stochastic delay control problems. The approximate controllability result is based on the controllability theory of deterministic control problems.

The paper is organized as follows. In Section 2, we introduce basic concepts and results. In Section 3, we prove the existence and uniqueness of mild solutions of problem (1) for linear fractional noises and nonlinear fractional noises. In Section 4, we study approximate controllability. In Section 5, we give conclusions.

\section{Preliminaries}

In this section, we introduce basic concepts and results.

\subsection{Fractional Brownian Motions}

Let $\left(\Sigma, \mathscr{F}, \mathbb{P}, \mathscr{F}_{t}\right)$ be a complete probability space with a filtration $\left\{\mathscr{F}_{t}\right\}_{t \in[0, \infty)}$ which satisfies usual conditions. A one-dimensional fractional Brownian motion $\left\{\beta^{H}(t)\right\}_{t \in[0, T]}$ is a centered Gaussian process which has zero mean and its covariance is

$$
\operatorname{Cov}(s, \tau)=\mathbb{E}\left[\beta^{H}(t) \beta^{H}(\tau)\right]=\frac{1}{2}\left(t^{2 H}+\tau^{2 H}-|t-\tau|^{2 H}\right) .
$$

Fractional Brownian motions can be expressed by Wiener processes, see [37].

Let $W$ be a real separable Hilbert space, $\left\{w_{i}\right\}_{i=1}^{\infty}$ be an orthonormal basis of $W$ and $\mathscr{L}(W)$ be the space of all bounded linear operators on $W$. Let $\mathcal{A} \in \mathscr{L}(W)$ be a symmetric, nonnegative operator and $\mathcal{A} w_{i}=\lambda_{i} w_{i}, i=1,2, \cdots$, with $\operatorname{tr} \mathcal{A}=\sum_{i=1}^{\infty} \lambda_{i}<\infty$. The infinite dimensional fractional Brownian motion $\left\{B_{\mathcal{A}}^{H}(t)\right\}_{t \in[0, T]}$ is defined by

$$
B_{\mathcal{A}}^{H}(t)=\sum_{i=1}^{\infty} \lambda_{i}^{1 / 2} w_{i} \beta_{i}^{H}(t)
$$

where $\left\{\beta_{i}^{H}\right\}_{i=1}^{\infty}$ is a sequence of independent one-dimensional fractional Brownian motions. $B_{\mathcal{A}}^{H}$ has zero mean and covariance $\operatorname{Cov}\left\langle B_{\mathcal{A}}^{H}(t), \mu\right\rangle\left\langle B_{\mathcal{A}}^{H}(\tau), v\right\rangle=\operatorname{Cov}(t, \tau)\langle\mathcal{A} \mu, v\rangle$, for any $t, \tau \in[0, T]$ and $\mu, v \in W$. In this paper, we consider the cylindrical fractional Brownian motion $B^{H}$, i.e., $\lambda_{i}=1, i=1,2, \cdots$. Let $\mathscr{L}_{2}^{0}\left(W, L^{2}(\mathcal{O})\right)$ be the space of Hilbert-Schmidt operators. An operator $\bar{g} \in \mathscr{L}_{2}^{0}\left(W, L^{2}(\mathcal{O})\right)$ satisfies $\bar{g} \in \mathscr{L}\left(W, L^{2}(\mathcal{O})\right)$ and

$$
\|\bar{g}\|_{\mathscr{L}_{2}^{0}}^{2}=\sum_{i=1}^{\infty}\left\|\bar{g} w_{i}\right\|_{L^{2}}^{2}<\infty
$$


Endowed the inner product $\langle\bar{g}, \tilde{g}\rangle_{\mathscr{L}_{2}^{0}}=\sum_{i=1}^{\infty}\left\langle\bar{g} w_{i}, \tilde{g} w_{i}\right\rangle_{L^{2}},\left(\mathscr{L}_{2}^{0}\left(W, L^{2}(\mathcal{O})\right),\langle\cdot, \cdot\rangle_{\mathscr{L}_{2}^{0}}\right)$ is a separable Hilbert space. We recall the following inequality:

Lemma 1 ([38]). Let $g:[0, T] \rightarrow \mathscr{L}_{2}^{0}\left(W, L^{2}(\mathcal{O})\right)$ and $\int_{0}^{T}\|g(s)\|_{\mathscr{L}_{2}^{0}}^{2} d s<\infty$, then stochastic integral $\int_{0}^{t} g(s) d B^{H}(s)$ is a well defined $L^{2}(\mathcal{O})$-valued random variable and satisfies

$$
\mathbb{E}\left\|\int_{0}^{t} g(s) d B^{H}(s)\right\|_{L^{2}}^{2} \leq 2 H t^{2 H-1} \int_{0}^{t}\|g(s)\|_{\mathscr{L}_{2}^{0}}^{2} d s, \forall t \in[0, T] .
$$

\subsection{Poisson Jumps}

Let $(U, \mathscr{B}(U), e)$ be a $\sigma$-finite measurable space and $\left\{\pi_{t}\right\}_{t \in[0, \infty)}$ be a stationary Poisson point process which is defined on $(\Sigma, \mathscr{F}, \mathbb{P})$ and take values in $U$. The compensated Poisson martingale measure $\tilde{\theta}$ is defined by

$$
\tilde{\theta}(d u, t)=\theta(d u, t)-e(d u) t, \forall t \in[0, \infty),
$$

where $\theta$ is a counting measure which is generated by $\left\{\pi_{t}\right\}_{t \in[0, \infty)}$. $\tilde{\theta}$ has zero mean and variance $\mathbb{E}[\tilde{\theta}(d u, t)]^{2}=t e(d u)$.

Let $M_{\mathscr{F}}^{e, 2}\left([0, T] \times U, L^{2}(\mathcal{O})\right)$ be the space of $\mathscr{F} \times \mathscr{B}(U)$ measurable processes with finite second moments and be equipped with the norm

$$
\|h\|_{M_{\mathscr{F}}^{e, 2}}^{2}=\mathbb{E} \int_{0}^{T} \int_{U}\|h(s, y ; u)\|_{L^{2}}^{2} e(d u) d s .
$$

If $h \in M_{\mathscr{F}}^{e, 2}\left([0, T] \times U, L^{2}(\mathcal{O})\right)$, then

$$
\int_{0}^{t} \int_{U} h(s, x, y ; u) \tilde{\theta}(d u, d s), \forall t \in[0, T]
$$

is a $L^{2}\left(\Sigma ; L^{2}(\mathcal{O})\right)$-valued random process which has zero mean. Furthermore, recall Theorem 6.1 in [39]:

Lemma 2 ([39]). If $h \in M_{\mathscr{F}}^{e, 2}\left([0, T] \times U, L^{2}(\mathcal{O})\right)$, then the isomorphic formula

$$
\mathbb{E}\left\|\int_{0}^{t} \int_{U} h(s, y ; u) \tilde{\theta}(d u, d s)\right\|_{L^{2}}^{2}=\mathbb{E} \int_{0}^{t} \int_{U}\|h(s, y ; u)\|_{L^{2}}^{2} e(d u) d s, \forall t \in[0, T],
$$

holds. Moreover, the following inequality holds

$$
\sup _{t \in[0, T]} \mathbb{E}\left\|\int_{0}^{t} \int_{U} h(s, y ; u) \tilde{\theta}(d u, d s)\right\|_{L^{2}}^{2} \leq 4 \mathbb{E} \int_{0}^{T} \int_{U}\|h(t, y ; u)\|_{L^{2}}^{2} e(d u) d t, \forall T>0 .
$$

\subsection{Fractional Differential Operators}

Consider the deterministic problem without time delay

$$
\left\{\begin{array}{l}
{ }_{0}^{C} D_{t}^{\alpha} y(t, x)+(-\Delta)^{\gamma} y(t, x)=f(t, x), t \in(0, \infty), x \in \mathbb{R}^{N}, \\
y(0, x)=\varphi(x), x \in \mathbb{R}^{N} .
\end{array}\right.
$$

The Riemann-Liouville kernel function is given by $R_{\alpha}(t)=\frac{t^{\alpha-1}}{\Gamma(\alpha)}, t, \alpha>0$, where $\Gamma$ denotes the Gamma function. Denote Laplace transform by $\mathcal{L}$, then

$$
\mathcal{L}\left[R_{\alpha}(t), \lambda\right]=\lambda^{-\alpha}, \alpha>0 .
$$


The $\alpha$ th Riemann-Liouville fractional integral is defined by $I^{\alpha} f(t)=\left(R_{\alpha} \star f\right)(t)$, where $\star$ denotes convolution. The $\alpha$ th Riemann-Liouville fractional derivative and Caputo fractional derivative are defined by

$$
{ }^{R} D_{t}^{\alpha} f(t)=\frac{d}{d t} I^{1-\alpha} f(t),{ }_{0}^{C} D_{t}^{\alpha} f(t)=\left(I^{1-\alpha} \frac{d}{d t} f\right)(t),
$$

respectively. The connection between ${ }^{R} D_{t}^{\alpha}$ and ${ }_{0}^{c} D_{t}^{\alpha}$ is given by

$$
{ }^{R} D_{t}^{\alpha}[f-f(0)](t)={ }_{0}^{C} D_{t}^{\alpha} f(t),
$$

for sufficiently smooth function $f$. There are several approaches to define the fractional Laplacian. We introduce the definition by means of Fourier transform $\mathcal{F}$,

$$
\mathcal{F}\left[(-\Delta)^{\gamma} f\right]=|\zeta|^{2 \gamma} \mathcal{F} f, \gamma \in(0,1) .
$$

Let the space

$$
H^{\gamma}(\mathcal{O})=\left\{f \in L^{2}\left(\mathbb{R}^{N}\right): \int_{\mathbb{R}^{N}}\left(1+|\xi|^{2 \gamma}\right)|\mathcal{F} f(\xi)|^{2} d \xi<\infty \text { and } u=0 \text {, a.e. in } \mathbb{R}^{N} \backslash \mathcal{O}\right\}
$$

be endowed the norm

$$
\|f\|_{H^{\gamma}(\mathcal{O})}=2 C(N, \gamma)^{-1} \int_{\mathbb{R}^{N}}|\xi|^{2 \gamma}|\mathcal{F} f(\xi)|^{2} d \xi,
$$

where $C(N, \gamma)$ is a constant dependent on $N$ and $\gamma$. Let $H_{0}^{\gamma}(\mathcal{O})$ denote the closure of $\mathrm{C}_{0}^{\infty}(\mathcal{O})$ in $H^{\gamma}(\mathcal{O})$. We recall the embedding result in [40].

Lemma 3 ([40]). Let $\gamma \in(0,1)$, the space $H_{0}^{\gamma}(\mathcal{O})$ is compactly embedded in $L^{2}(\mathcal{O})$, and

$$
\|f\|_{2} \leq C\|f\|_{H_{0}^{\gamma}}
$$

Fractional Laplacian can be extended to $H_{0}^{\gamma}(\mathcal{O})$, i.e., $(-\Delta)^{\gamma}: H_{0}^{\gamma}(\mathcal{O}) \rightarrow L^{2}(\mathcal{O})$. Let $\left\{S_{\gamma}(t)\right\}_{t \geq 0}$ be the semigroup generated by $(-\Delta)^{\gamma}$. Then $\left\{S_{\gamma}(t)\right\}_{t \geq 0}$ is a $C_{0}$-contraction semigroup on $L^{2}(\mathcal{O})$. On the other hand, since the embedding from $H_{0}^{\gamma}(\mathcal{O})$ to $L^{2}(\mathcal{O})$ is compact, we get that

Proposition 1. $S_{\gamma}(t): L^{2}(\mathcal{O}) \rightarrow L^{2}(\mathcal{O})$ is a compact operator, for any $t \geq 0$.

The Mittag-Leffler function $\mathcal{E}_{k_{1}, k_{2}}$ and the Mainardi function $\mathcal{M}_{k}$ are defined by

$$
\begin{array}{r}
\mathcal{E}_{k_{1}, k_{2}}(z)=\sum_{n=0}^{\infty} \frac{z^{n}}{\Gamma\left(n k_{1}+k_{2}\right)}, \text { for } k_{1}, k_{2}>0, z \in \mathbb{C}, \\
\mathcal{M}_{k}(z)=\sum_{n=0}^{\infty} \frac{(-z)^{n}}{n ! \Gamma(-n k+1-k)}, \text { for } k \in(0,1), z \in \mathbb{C},
\end{array}
$$

respectively. Then $\mathcal{L}\left[\mathcal{M}_{k}, \lambda\right]=\mathcal{E}_{k, 1}(-\lambda)$. Let

$$
Y_{\gamma}^{\alpha}(t)=\int_{0}^{\infty} \mathcal{M}_{\alpha}(s) S_{\gamma}\left(s t^{\alpha}\right) d s, T_{\gamma}^{\alpha}(t)=\alpha \int_{0}^{\infty} s \mathcal{M}_{\alpha}(s) S_{\gamma}\left(s t^{\alpha}\right) d s
$$

and $Z_{\gamma}^{\alpha}(t)=t^{\alpha-1} T_{\gamma}^{\alpha}(t)$, for all $t>0$. By Laplace transform in time variable and Fourier transform in spatial variable on both sides of the equation in (5), the formal solution of problem (5) is given by

$$
y(t)=Y_{\gamma}^{\alpha}(t) \varphi+\int_{0}^{t} Z_{\gamma}^{\alpha}(t-s) f(s) d s
$$


Furthermore, denote

$$
Y_{\gamma}^{\alpha}(t) y(x)=\eta(t, \cdot) \star y(x), Z_{\gamma}^{\alpha}(t) y(x)=\xi(t, \cdot) \star y(x) .
$$

Then, equivalent to (6), the formal solution $y$ satisfies

$$
y(t, x)=\int_{\mathbb{R}^{N}} \eta(t, x-z) \varphi(z) d z+\int_{0}^{t} \int_{\mathbb{R}^{N}} \xi(t-s, x-z) f(s, z) d z d s .
$$

Recall the properties of $Y_{\gamma}^{\alpha}, Z_{\gamma}^{\alpha}, \eta$ and $\xi$. Let

$$
\lambda_{1}=\left\{\begin{array}{ll}
\frac{N}{N-2 \gamma}, & N>2 \gamma, \\
\infty, & \text { otherwise }
\end{array} \quad \lambda_{2}= \begin{cases}\frac{N}{N-4 \gamma}, & N>4 \gamma \\
\infty, & \text { otherwise }\end{cases}\right.
$$

Lemma 4 ([20]). Assume $\alpha \in(0,1), \gamma \in(1 / 2,1)$. Then

1. Let $q \in\left[1, \lambda_{1}\right)$, then $\eta(t, \cdot) \in C\left((0, \infty), L^{q}(\mathcal{O})\right)$ and

$$
\|\eta(t, \cdot)\|_{q} \leq C t^{-\frac{\alpha N}{2 \gamma}\left(1-\frac{1}{q}\right)}
$$

2. Let $q \in\left[1, \lambda_{2}\right)$, then $\xi(t, \cdot) \in C\left((0, \infty), L^{q}(\mathcal{O})\right)$ and

$$
\|\xi(t, \cdot)\|_{q} \leq C t^{-\frac{\alpha N}{2 \gamma}\left(1-\frac{1}{q}\right)+\alpha-1}
$$

Let

$$
\theta_{1}=\left\{\begin{array}{ll}
\frac{2 N}{N-4 \gamma}, & N>4 \gamma, \\
\infty, & \text { otherwise }
\end{array} \quad \theta_{2}= \begin{cases}\frac{2 N}{N-8 \gamma}, & N>8 \gamma \\
\infty, & \text { otherwise }\end{cases}\right.
$$

Lemma 5 ([20]). Assume $\alpha \in(0,1), \gamma \in(1 / 2,1)$. Then

1. $Y_{\gamma}^{\alpha}$ and $Z_{\gamma}^{\alpha}$ satisfy

$$
\left\|Y_{\gamma}^{\alpha}(t) y\right\|_{\infty} \leq\|y\|_{\infty},\left\|Z_{\gamma}^{\alpha}(t) y\right\|_{\infty} \leq \frac{1}{\Gamma(\alpha)} t^{\alpha-1}\|y\|_{\infty}
$$

2. For $p \in\left[1, \theta_{1}\right)$ and $q \in\left[1, \theta_{2}\right)$,

$$
\left\|Y_{\gamma}^{\alpha}(t) y\right\|_{p} \leq C t^{-\frac{\alpha N}{2 \gamma}\left(\frac{1}{2}-\frac{1}{p}\right)}\|y\|_{2},\left\|Z_{\gamma}^{\alpha}(t) y\right\|_{q} \leq C t^{-\frac{\alpha N}{2 \gamma}\left(\frac{1}{2}-\frac{1}{q}\right)+\alpha-1}\|y\|_{2} .
$$

3. Let $\kappa \in[1, \infty)$ and $y \in L^{\kappa}(\mathcal{O})$, then the map $t \mapsto Y_{\gamma}^{\alpha}(t)$ y belongs to $C\left([0, \infty), L^{\kappa}(\mathcal{O})\right)$.

\subsection{Function Spaces}

Let $L^{2}\left(\Sigma ; L^{2}(\mathcal{O})\right)$ be the set of all $\mathscr{F}$ measurable random variables $\zeta$ such that

$$
\|\zeta\|_{L^{2}}^{2}=\mathbb{E}\|\zeta\|_{2}^{2}<\infty
$$

Let $C_{\mathcal{F}_{0}} \doteq C_{\mathcal{F}_{0}}\left([-\tau, 0] ; L^{2}(\mathcal{O})\right)$ be the space of initial states. We say $\varphi \in C_{\mathcal{F}_{0}}\left([-\tau, 0] ; L^{2}(\mathcal{O})\right)$ if $\varphi$ is $\mathcal{F}_{0}$-adapted and continuous from $[-\tau, 0]$ to $L^{2}(\mathcal{O})$, a.s., with

$$
\|\varphi\|_{\mathcal{C}_{\mathcal{F}_{0}}}^{2}=\sup _{t \in[-\tau, 0]} \mathbb{E}\|\varphi(t, \cdot)\|_{2}^{2}<\infty
$$

We use notation $L_{\mathscr{F} t}^{2}\left([-\tau, T] ; H_{0}^{\gamma}(\mathcal{O})\right)$ to denote the space of all $\mathcal{F}_{t}$-adapted random processes such that

$$
\|y\|_{L_{\mathscr{F}_{t}}^{2}}^{2}=\mathbb{E} \int_{-\tau}^{T}\|y(t, \cdot)\|_{H_{0}^{\gamma}}^{2} d t<\infty
$$


Let $C_{\mathscr{F}_{t}} \doteq C_{\mathscr{F}_{t}}\left([-\tau, T] ; L^{2}(\mathcal{O})\right)$ be the space of all continuous random processes from $[-\tau, T]$ to $L^{2}(\mathcal{O})$, a.s., with essentially finite second moments and be endowed with the following norm

$$
\|y\|_{C_{\mathscr{F}_{t}}}^{2}=\sup _{t \in[0, T]} \mathbb{E}\|y(t, \cdot)\|_{2}^{2}+\left\|\left.y\right|_{[-\tau, 0]}\right\|_{\mathcal{C}_{\mathcal{F}_{0}}}^{2}
$$

Let $\mathscr{D}(T)$ be the set of all stochastic processes with the following properties:

1. $y \in L_{\mathscr{F}_{t}}^{2}\left([-\tau, T] ; H_{0}^{\gamma}(\mathcal{O})\right) \cap C_{\mathscr{F}_{t}}\left([-\tau, T] ; L^{2}(\mathcal{O})\right)$;

2. $y(t, x)=\varphi(t, x)$, a.s., for all $(t, x) \in[-\tau, 0] \times \mathcal{O}$;

3. $y(t, x)=0$, a.s., for all $(t, x) \in[-\tau, T] \times \mathbb{R}^{N} \backslash \mathcal{O}$.

Then the definition of mild solutions is given by

Definition 1. We call $y \in \mathscr{D}(T)$ a mild solution of problem (1) if for any $t \in[0, T], y$ satisfies the following formula

$$
\begin{aligned}
y= & Y_{\gamma}^{\alpha}(t) \varphi(0)+\int_{0}^{t} Z_{\gamma}^{\alpha}(t-s) f\left(s, y_{\tau}\right) d s+\int_{0}^{t} Z_{\gamma}^{\alpha}(t-s) \mathcal{B} v(s) d s \\
& +\int_{0}^{t} Z_{\gamma}^{\alpha}(t-s) g\left(s, y_{\tau}\right) d B^{H}(s)+\int_{0}^{t} \int_{U} Z_{\gamma}^{\alpha}(t-s) h\left(s, y_{\tau} ; u\right) \tilde{\theta}(d u, d s), \text { a.s. }
\end{aligned}
$$

or equivalently,

$$
\begin{aligned}
y(t, x)= & \int_{\mathcal{O}} \eta(t, x-z) \varphi(0, z) d z+\int_{0}^{t} \int_{\mathcal{O}} \xi(t-s, x-z) \mathcal{B} v(s)(z) d z d s \\
& +\int_{0}^{t} \int_{\mathcal{O}} \xi(t-s, x-z) f\left(s, z, y_{\tau}(s, z)\right) d z d s \\
& +\int_{0}^{t} \int_{\mathcal{O}} \xi(t-s, x-z) g\left(s, y_{\tau}(s, z)\right) d z d B^{H}(s) \\
& +\int_{0}^{t} \int_{U} \int_{\mathcal{O}} \xi(t-s, x-z) h\left(s, z, y_{\tau}(s, z) ; u\right) d z \tilde{\theta}(d u, d s), \text { a.s., }
\end{aligned}
$$

if each integral is well defined.

Remark 1. Similar to the proof in [41], we can prove that a classic solution is also a mild solution defined in the sense of Definition 1.

\section{Mild Solutions}

In this section, we prove the existence and uniqueness of the mild solution of problem (1) with linear fractional noise and nonlinear fractional noise by approximate method and the Banach Fixed Point Theorem.

We propose the following hypotheses on nonlinear functions $f$ and $h$ :

Hypothesis 1 (H1). For any $t \in[0, T]$ and $x \in \mathcal{O}, h \in M_{\mathcal{F}}^{e, 2}\left([0, T] \times U, L^{2}(\mathcal{O})\right)$ satisfies

$$
\begin{gathered}
\int_{U}|h(t, x, y(t, x) ; u)|^{2} e(d u) \leq M_{1}\left(1+|y(t, x)|^{2}\right), \\
\int_{U}|h(t, x, \tilde{y}(t, x) ; u)-h(t, x, \hat{y}(t, x) ; u)|^{2} e(d u) \leq L_{1}|\tilde{y}(t, x)-\hat{y}(t, x)|^{2},
\end{gathered}
$$

where constants $M_{1}, L_{1}>0$.

Hypothesis 2 (H2). For any $t \in[0, T]$ and $x \in \mathcal{O}$, there exist a positive function $\Phi \in L^{1}(\mathcal{O})$ and a constant $M_{2}>0$ such that

$$
\int_{0}^{t}|f(s, x, 0)|^{2} d s \leq \Phi(x)
$$




$$
\int_{0}^{t}|f(s, x, \tilde{y}(s, x))-f(s, x, \hat{y}(s, x))|^{2} d s \leq M_{2} \int_{-\tau}^{t}|\tilde{y}(s, x)-\hat{y}(s, x)|^{2} d s .
$$

\subsection{Linear Fractional Noises}

In this subsection, we discuss the following problem:

$$
\left\{\begin{aligned}
&{ }_{0}^{C} D_{t}^{\alpha} y(t, x)+(-\Delta)^{\gamma} y(t, x)=f\left(t, x, y_{\tau}(t, x)\right)+\mathcal{B} v(t)(x)+g(t) \frac{d B^{H}(t)}{d t} \\
& \quad+\int_{U} h\left(t, x, y_{\tau}(t, x) ; u\right) \tilde{\theta}(d u), t \in(0, T], x \in \mathcal{O}, \\
& y(t, x)=\varphi(t, x), \text { a.s., } t \in[-\tau, 0], x \in \mathcal{O}, \\
& y(t, x)=0, \text { a.s., } t \in[-\tau, T], x \in \mathbb{R}^{N} \backslash \mathcal{O} .
\end{aligned}\right.
$$

Assume that

Hypothesis 3 (H3). $g:[0, T] \rightarrow \mathscr{L}_{2}^{0}\left(W, L^{2}(\mathcal{O})\right)$ satisfies

$$
\|g(t)\|_{\mathscr{L}_{2}^{0}}^{2} \leq M_{3}, \forall t \in[0, T]
$$

where constant $M_{3}>0$.

The main result of this subsection is as follows.

Theorem 1. Let $1 / 2<\alpha, \gamma, H<1, N>2 \gamma$, (H1), (H2), and (H3) hold. If $\varphi \in C_{\mathscr{F}_{0}}\left([-\tau, 0] ; L^{2}(\mathcal{O})\right)$ and $v \in L^{2}([0, T] ; V)$, then there exists a unique mild solution $y \in \mathscr{D}(T)$ of the problem (9).

Proof. We use the approximate strategy to prove the result. The proof is divided into four parts. First, we consider the regularity of stochastic integrals. Second, we construct a sequence $\left\{y^{n}\right\}_{n=1}^{\infty}$ to approximate the solution of problem (9). Third, we show that the limit of $\left\{y^{n}\right\}_{n=1}^{\infty}$ in $\mathscr{D}(T)$ is a mild solution of problem (9). At last, we prove the uniqueness of mild solutions by the Gronwall inequality.

Step 1: The regularity of stochastic integrals. (i) We show that $\int_{0}^{t} \int_{\mathcal{O}} \xi(t-s, x-$ $z) g(s) d z d B^{H}(s) \in C_{\mathscr{F}_{t}}\left([0, T] ; L^{2}(\mathcal{O})\right)$. For arbitrary $\epsilon>0$, consider

$$
\begin{aligned}
& \mathbb{E}\left\|\int_{0}^{t+\epsilon} \int_{\mathcal{O}} \xi(t-s+\epsilon, x-z) g(s) d z d B^{H}(s)-\int_{0}^{t} \int_{\mathcal{O}} \xi(t-s, x-z) g(s) d z d B^{H}(s)\right\|_{2}^{2} \\
\leq & 2 \mathbb{E}\left\|\int_{t}^{t+\epsilon} \int_{\mathcal{O}} \xi(t-s+\epsilon, x-z) g(s) d z d B^{H}(s)\right\|_{2}^{2} \\
& +2 \mathbb{E}\left\|\int_{0}^{t} \int_{\mathcal{O}}[\xi(t-s+\epsilon, x-z)-\xi(t-s, x-z)] g(s) d z d B^{H}(s)\right\|_{2}^{2} \\
\doteq & J_{1}+J_{2} .
\end{aligned}
$$

By Lemma 1, the Young inequality, (H3) and Lemma 4, we deduce that

$$
\begin{aligned}
J_{1} & \leq 4 H \epsilon^{2 H-1} \int_{t}^{t+\epsilon}\left\|\int_{\mathcal{O}} \xi(t-s+\epsilon, x-z) g(s) d z\right\|_{\mathscr{L}_{2}^{0}}^{2} d s \\
& \leq 4 H \epsilon^{2 H-1} \int_{t}^{t+\epsilon}\|\xi(t-s+\epsilon, \cdot)\|_{1}^{2}\|g(s)\|_{\mathscr{L}_{2}^{0}}^{2} d s \\
& \leq 4 C H \epsilon^{2 H-1} \int_{t}^{t+\epsilon}(t-s+\epsilon)^{2 \alpha-2}\|g(s)\|_{\mathscr{L}_{2}^{0}}^{2} d s \\
& \leq 4 C H M_{3} \epsilon^{2 H+2 \alpha-2} \\
& \rightarrow 0
\end{aligned}
$$


as $\epsilon \rightarrow 0$, since $\alpha+H-1>0$. Given any constant $\lambda>0$ which is independent on $\epsilon$, we consider

$$
\begin{aligned}
J_{2} \leq & 4 M_{3} H T^{2 H-1} \int_{0}^{t-\lambda}\|\xi(t-s+\epsilon, \cdot)-\xi(t-s, \cdot)\|_{1}^{2} d s \\
& +4 M_{3} H T^{2 H-1} \int_{t-\lambda}^{t}\|\xi(t-s+\epsilon, \cdot)-\xi(t-s, \cdot)\|_{1}^{2} d s .
\end{aligned}
$$

Based on Lemma $4, \xi$ is continuous on $(0, \infty)$, then $\xi$ is uniformly continuous on $[\lambda, T]$. Hence,

$$
4 M_{3} H T^{2 H-1} \int_{0}^{t-\lambda}\|\xi(t-s+\epsilon, \cdot)-\xi(t-s, \cdot)\|_{1}^{2} d s \rightarrow 0 \text { as } \epsilon \rightarrow 0 .
$$

On the other hand,

$$
\begin{aligned}
& 4 M_{3} H T^{2 H-1} \int_{t-\lambda}^{t}\|\xi(t-s+\epsilon, \cdot)-\xi(t-s, \cdot)\|_{1}^{2} d s \\
\leq & 8 C M_{3} H T^{2 H-1} \int_{t-\lambda}^{t}(t-s+\epsilon)^{2 \alpha-2}+(t-s)^{2 \alpha-2} d s \\
\leq & 8 C M_{3} H T^{2 H-1}\left[\epsilon^{2 \alpha-1}-(\lambda+\epsilon)^{2 \alpha-1}+\lambda^{2 \alpha-1}\right] \\
\rightarrow & 0,
\end{aligned}
$$

as $\lambda \rightarrow 0$. Therefore, $J_{2} \rightarrow 0$ as $\epsilon \rightarrow 0$. We conclude that $\int_{0}^{t} \int_{\mathcal{O}} \xi(t-s, x-z) g(s) d z d B^{H}(s) \in$ $C_{\mathscr{F}_{t}}\left([0, T] ; L^{2}(\mathcal{O})\right)$.

(ii) We illustrate that $\int_{0}^{t} \int_{U} \int_{\mathcal{O}} \xi(t-s, x-z) h\left(s, z, y_{\tau}(s, z) ; u\right) d z \tilde{\theta}(d u, d s)$ is continuous on $[0, T]$. First, for any $\epsilon>0$, we consider

$$
\begin{aligned}
\mathbb{E} \| & \int_{0}^{t+\epsilon} \int_{U} \int_{\mathcal{O}} \xi(t-s+\epsilon, x-z) h\left(s, z, y_{\tau}(s, z) ; u\right) d z \tilde{\theta}(d u, d s) \\
& \quad-\int_{0}^{t} \int_{U} \int_{\mathcal{O}} \xi(t-s, x-z) h\left(s, z, y_{\tau}(s, z) ; u\right) d z \tilde{\theta}(d u, d s) \|_{2}^{2} \\
\leq & 2 \mathbb{E}\left\|\int_{0}^{t} \int_{U} \int_{\mathcal{O}}[\xi(t-s+\epsilon, x-z)-\xi(t-s, x-z)] h\left(s, z, y_{\tau}(s, z) ; u\right) d z \tilde{\theta}(d u, d s)\right\|_{2}^{2} \\
& +2 \mathbb{E}\left\|\int_{t}^{t+\epsilon} \int_{U} \int_{\mathcal{O}} \xi(t-s+\epsilon, x-z) h\left(s, z, y_{\tau}(s, z) ; u\right) d z \tilde{\theta}(d u, d s)\right\|_{2}^{2} \\
\doteq J_{3}+J_{4} . &
\end{aligned}
$$

By Lemma 2, the Young inequality, Lemma 4 and (H1) we get that

$$
\begin{aligned}
& J_{4} \leq 2 \mathbb{E} \int_{t}^{t+\epsilon} \int_{U}\|\xi(t-s+\epsilon, \cdot)\|_{1}^{2}\left\|h\left(s, \cdot, y_{\tau}(s, \cdot) ; u\right)\right\|_{2}^{2} e(d u) d s \\
& \leq 2 \mathrm{CE} \int_{t}^{t+\epsilon} \int_{U}(t-s+\epsilon)^{2 \alpha-2}\left\|h\left(s, \cdot, y_{\tau}(s, \cdot) ; u\right)\right\|_{2}^{2} e(d u) d s \\
& \leq 2 C M_{1} \mathbb{E} \int_{t}^{t+\epsilon}(t-s+\epsilon)^{2 \alpha-2} \int_{\mathcal{O}}\left(1+\left|y_{\tau}(s, z)\right|^{2}\right) d z d s \\
& \leq 2 C M_{1}|\mathcal{O}| \int_{t}^{t+\epsilon}(t-s+\epsilon)^{2 \alpha-2} d s+2 C M_{1} \mathbb{E} \int_{t}^{t+\epsilon}(t-s+\epsilon)^{2 \alpha-2} \int_{\mathcal{O}}\left|y_{\tau}(s, z)\right|^{2} d z d s \\
& \leq 2 C M_{1}|\mathcal{O}| \epsilon^{2 \alpha-1}+2 C M_{1}\|y\|_{C_{\mathscr{F}_{t}}}^{2} \epsilon^{2 \alpha-1} \text {. }
\end{aligned}
$$

Since $1 / 2<\alpha<1$, it is obvious that $J_{4} \rightarrow 0$ as $\epsilon \rightarrow 0$. 
By similar arguments to $J_{4}$ and $J_{2}$, for any $\lambda>0$, we obtain

$$
\begin{aligned}
J_{3} & \leq C M_{1}\left(1+\|y\|_{\mathscr{C}_{\mathscr{F}_{t}}}^{2}\right) \int_{0}^{t}\|\xi(t-s+\epsilon, \cdot)-\xi(t-s, \cdot)\|_{1}^{2} d s \\
& \leq C M_{1}\left(1+\|y\|_{\mathscr{C}_{F_{t}}}^{2}\right)\left\{\int_{0}^{t-\lambda}+\int_{t-\lambda}^{t}\right\}\|\xi(t-s+\epsilon, \cdot)-\xi(t-s, \cdot)\|_{1}^{2} d s \\
& \rightarrow 0 \text { as } \epsilon \rightarrow 0 .
\end{aligned}
$$

Thus, we summarize that

$$
\int_{0}^{t} \int_{U} \int_{\mathcal{O}} \xi(t-s, x-z) h\left(s, z, y_{\tau}(s, z) ; u\right) d z \tilde{\theta}(d u, d s) \in C_{\mathscr{F}_{t}}\left([0, T] ; L^{2}(\mathcal{O})\right) .
$$

Step 2: Approximate solutions. Let

$$
\begin{aligned}
y^{1}(t, x)= & \int_{\mathcal{O}} \eta(t, x-z) \varphi(0, z) d z, \\
y^{n+1}(t, x)= & y^{1}(t, x)+\int_{0}^{t} \int_{\mathcal{O}} \xi(t-s, x-z) f\left(s, z, y_{\tau}^{n}(s, z)\right) d z d s \\
& +\int_{0}^{t} \int_{\mathcal{O}} \xi(t-s, x-z) \mathcal{B} v(s)(z) d z d s+\int_{0}^{t} \int_{\mathcal{O}} \xi(t-s, x-z) g(s) d z d B^{H}(s) \\
& +\int_{0}^{t} \int_{U} \int_{\mathcal{O}} \xi(t-s, x-z) h\left(s, z, y_{\tau}^{n}(s, z) ; u\right) d z \tilde{\theta}(d u, d s) \\
\doteq & y^{1}(t, x)+K_{1}(t, x)+K_{2}(t, x)+K_{3}(t, x)+K_{4}(t, x)
\end{aligned}
$$

with $y^{n}(t, x)=\varphi(t, x)$, a.s., for all $t \in[-\tau, 0]$ and $x \in \mathcal{O}, n=1,2, \cdots$. Next, we show that $\left\{y^{n}\right\}_{n=1}^{\infty}$ is a sequence in $\mathcal{D}(T)$. It is clear that $y^{1}(t, x) \in \mathcal{D}(T)$ by Lemma 5. Let $y^{n}, n \geq 2$, belong to $\mathcal{D}(T)$. We show that $y^{n+1}$ belongs to $\mathcal{D}(T)$. Since we have already proved the regularity of $K_{3}(t, x)$ and $K_{4}(t, x)$ in Step 1 , then we remain to prove that $K_{1}(t, x)$ and $K_{2}(t, x)$ belong to $\mathcal{D}(T)$.

We show that $K_{1}(t, x) \in C_{\mathscr{F}_{t}}\left([0, T] ; L^{2}(\mathcal{O})\right)$. For any $\epsilon>0$, we consider

$$
\begin{aligned}
& \mathbb{E} \| \int_{0}^{t+\epsilon} \int_{\mathcal{O}} \xi(t-s+\epsilon, x-z) f\left(s, z, y_{\tau}^{n}(s, z)\right) d z d s \\
& \quad-\int_{0}^{t} \int_{\mathcal{O}} \xi(t-s, x-z) f\left(s, z, y_{\tau}^{n}(s, z)\right) d z d s \|_{2}^{2} \\
\leq & \mathbb{E}\left\|\int_{0}^{t} \int_{\mathcal{O}}[\xi(t-s+\epsilon, x-z)-\xi(t-s, x-z)] f\left(s, z, y_{\tau}^{n}(s, z)\right) d z d s\right\|_{2}^{2} \\
& +\mathbb{E}\left\|\int_{t}^{t+\epsilon} \int_{\mathcal{O}} \xi(t-s+\epsilon, x-z) f\left(s, z, y_{\tau}^{n}(s, z)\right) d z d s\right\|_{2}^{2} \\
\doteq & J_{5}+J_{6} .
\end{aligned}
$$
obtain

By the Minkowsky inequality, the Young inequality and the Hölder inequality, we

$$
\begin{aligned}
J_{5} & \leq \mathbb{E}\left\{\int_{0}^{t}\left\|\int_{\mathcal{O}}[\xi(t-s+\epsilon, x-z)-\xi(t-s, x-z)] f\left(s, z, y_{\tau}^{n}(s, z)\right) d z\right\|_{2} d s\right\}^{2} \\
& \leq \mathbb{E}\left\{\int_{0}^{t}\|\xi(t-s+\epsilon, \cdot)-\xi(t-s, \cdot)\|_{1}\left\|f\left(s, \cdot, y_{\tau}^{n}(s, \cdot)\right)\right\|_{2} d s\right\}^{2} \\
& \leq \int_{0}^{t}\|\xi(t-s+\epsilon, \cdot)-\xi(t-s, \cdot)\|_{1}^{2} d s \mathbb{E} \int_{0}^{t}\left\|f\left(s, \cdot, y_{\tau}^{n}(s, \cdot)\right)\right\|_{2}^{2} d s .
\end{aligned}
$$


On the other hand, by the Fubini Lemma and (H2) we get

$$
\begin{aligned}
& \mathbb{E} \int_{0}^{t}\left\|f\left(s, \cdot, y_{\tau}^{n}(s, \cdot)\right)\right\|_{2}^{2} d s \\
\leq & \mathbb{E} \int_{\mathcal{O}} \int_{0}^{T}\left|f\left(s, x, y_{\tau}^{n}(s, x)\right)-f(s, x, 0)+f(s, x, 0)\right|^{2} d s d x \\
\leq & 2\|\Phi\|_{L^{1}}+2 M_{2} \mathbb{E} \int_{-\tau}^{t}\left\|y^{n}(s, \cdot)\right\|_{2}^{2} d s \\
\leq & C M_{2}\left(1+\left\|y^{n}\right\|_{\mathcal{F F}_{t}}^{2}\right) .
\end{aligned}
$$

Therefore,

$$
J_{5} \leq C M_{2}\left(1+\left\|y^{n}\right\|_{\mathscr{C}_{F_{t}}}^{2}\right) \int_{0}^{t}\|\xi(t-s+\epsilon, \cdot)-\xi(t-s, \cdot)\|_{1}^{2} d s \rightarrow 0 \text { as } \epsilon \rightarrow 0 .
$$

Further, similar to the proof of $J_{4}$, we obtain

$$
J_{6} \leq C M_{2}\left(1+\left\|y^{n}\right\|_{\mathscr{C}_{\mathscr{F}_{t}}}^{2}\right) \epsilon^{2 \alpha-1} \rightarrow 0 \text { as } \epsilon \rightarrow 0 .
$$

Thus, $K_{1}(t, x) \in C_{\mathscr{F}_{t}}\left([0, T] ; L^{2}(\mathcal{O})\right)$. By similar discussions to $K_{1}(t, x)$, we get that

$$
\begin{aligned}
& \mathbb{E}\left\|\int_{0}^{t+\epsilon} \int_{\mathcal{O}} \xi(t-s+\epsilon, x-z) \mathcal{B} v(s)(z) d z d s-\int_{0}^{t} \int_{\mathcal{O}} \xi(t-s, x-z) \mathcal{B} v(s)(z) d z d s\right\|_{2}^{2} \\
\leq & \int_{0}^{t}\|\xi(t-s+\epsilon, \cdot)-\xi(t-s, \cdot)\|_{1}^{2} d s \int_{0}^{t}\|\mathcal{B} v(s)\|_{2}^{2} d s+C \epsilon^{2 \alpha-1} \int_{0}^{t}\|\mathcal{B} v(s)\|_{2}^{2} d s \\
\leq & C\|v\|_{L^{2}([0, T], V)}^{2}\left\{\int_{0}^{t}\|\xi(t-s+\epsilon, \cdot)-\xi(t-s, \cdot)\|_{1}^{2} d s+\epsilon^{2 \alpha-1}\right\} \\
\rightarrow & 0 \text { as } \epsilon \rightarrow 0,
\end{aligned}
$$

i.e., $K_{2}(t, x) \in C_{\mathscr{F}_{t}}\left([0, T] ; L^{2}(\mathcal{O})\right)$. To sum up, for arbitrarily $\epsilon>0$,

$$
\begin{aligned}
& \mathbb{E}\left\|y^{n}(t+\epsilon, \cdot)-y^{n}(t, \cdot)\right\|_{2}^{2} \\
\leq & 16 \mathbb{E}\left\|y^{1}(t+\varepsilon, \cdot)-y^{1}(t, \cdot)\right\|_{2}^{2}+16 \sum_{i=1}^{4} \mathbb{E}\left\|K_{i}(t+\varepsilon, \cdot)-K_{i}(t, \cdot)\right\|_{2}^{2} \\
\rightarrow & 0 \text { as } \epsilon \rightarrow 0 .
\end{aligned}
$$

Therefore, $y^{n+1} \in \mathcal{D}(T)$. We conclude that $y^{n} \in \mathcal{D}(T)$ for $n=1,2, \cdots$.

Step 3: Existence of solutions. First, we show that $\left\{y^{n}\right\}_{n=1}^{\infty}$ is a convergent sequence in $\mathcal{D}(T)$. Similar discussions to $J_{4}$ and $J_{5}$, by $(\mathrm{H} 1)$ and $(\mathrm{H} 2)$, we obtain

$$
\begin{aligned}
& \quad \mathbb{E}\left\|y^{n+1}(t, \cdot)-y^{n}(t, \cdot)\right\|_{2}^{2} \\
& \leq 2 T^{2 \alpha-1} \mathbb{E} \int_{0}^{t} \int_{\mathcal{O}}\left|f\left(s, z, y_{\tau}^{n}(s, z)\right)-f\left(s, z, y_{\tau}^{n-1}(s, z)\right)\right|^{2} d z d s \\
& +2 \mathbb{E} \int_{0}^{t} \int_{U}\|\xi(t-s, \cdot)\|_{1}^{2} \int_{\mathcal{O}}\left|h\left(s, z, y_{\tau}^{n}(s, z) ; u\right)-h\left(s, z, y_{\tau}^{n-1}(s, z) ; u\right)\right|^{2} d z e(d u) d s \\
& \leq 4 T^{2 \alpha-1}\left(M_{2}+L_{1}\right) \int_{-\tau}^{t} \mathbb{E}\left\|y^{n}(s, \cdot)-y^{n-1}(s, \cdot)\right\|_{2}^{2} d s, n \geq 2 . \\
& \text { Let } \Psi_{n}(t)=\sup _{s \in[0, t]} \mathbb{E}\left\|y^{n+1}(s, \cdot)-y^{n}(s, \cdot)\right\|_{2}^{2} \text {, then we get } \\
& \qquad \Psi_{n}(t) \leq C\left(T, M_{2}, L_{1}, \alpha\right) \int_{-\tau}^{t} \Psi_{n-1}(s) d s, n=2,3, \cdots,
\end{aligned}
$$


for any $t \in[0, T]$. Moreover, $\Psi_{n}$ satisfies

$$
\Psi_{n}(t) \leq \frac{\left[C\left(T, M_{2}, L_{1}, \alpha\right)\right]^{n-1}}{(n-1) !} \Psi_{1}(T), n=2,3, \cdots, \forall t \in[0, T],
$$

by recurrence formula. Hence, $\left\{y^{n}\right\}_{n=1}^{\infty}$ is a Cauchy sequence in $C_{\mathscr{F}_{t}}\left([-\tau, T] ; L^{2}(\mathcal{O})\right)$. Thus, there exists an element $y \in C_{\mathscr{F}_{t}}\left([-\tau, T] ; L^{2}(\mathcal{O})\right)$ such that

$$
y^{n} \rightarrow y \text { as } n \rightarrow \infty \text { in } C_{\mathscr{F}_{t}}\left([-\tau, T] ; L^{2}(\mathcal{O})\right) .
$$

We assert that $y$ is a mild solution of problem (9). Consider

$$
\begin{aligned}
& \mathbb{E} \| y^{n+1}(t, x)-\int_{\mathcal{O}} \eta(t, x-z) \varphi(0, z) d z+\int_{0}^{t} \int_{\mathcal{O}} \xi(t-s, x-z) f\left(s, z, y_{\tau}(s, z)\right) d z d s \\
& \quad-\int_{0}^{t} \int_{\mathcal{O}} \xi(t-s, x-z) \mathcal{B} v(s)(z) d z d s+\int_{0}^{t} \int_{\mathcal{O}} \xi(t-s, x-z) g(s) d z d B^{H}(s) \\
& \quad-\int_{0}^{t} \int_{U} \int_{\mathcal{O}} \xi(t-s, x-z) h\left(s, z, y_{\tau}(s, z) ; u\right) d z \tilde{\theta}(d u, d s) \|_{2}^{2} \\
= & \mathbb{E} \| \int_{0}^{t} \int_{\mathcal{O}} \xi(t-s, x-z)\left[f\left(s, z, y_{\tau}^{n}(s, z)\right)-f\left(s, z, y_{\tau}(s, z)\right)\right] d z d s \\
& +\int_{0}^{t} \int_{U} \int_{\mathcal{O}} \xi(t-s, x-z)\left[h\left(s, z, y_{\tau}^{n}(s, z) ; u\right)-h\left(s, z, y_{\tau}(s, z) ; u\right) d z \tilde{\theta}(d u, d s) \|_{2}^{2}\right. \\
\leq & 4 T^{2 \alpha-1}\left(M_{2}+L_{1}\right) \int_{-\tau}^{T} \mathbb{E}\left\|y^{n}(s, \cdot)-y(s, \cdot)\right\|_{2}^{2} d s \\
\rightarrow & 0,
\end{aligned}
$$

as $n \rightarrow \infty$. The assertion is proved, i.e., $y$ is a mild solution to the problem (9).

Step 4: Uniqueness of solutions. Assume that $\tilde{y}, \bar{y} \in \mathscr{D}(T)$ are two different mild solutions of problem (9) with same initial state $\varphi$ and control $v$. By similar discussions to (11), we get

$$
\mathbb{E}\|\tilde{y}(t, \cdot)-\bar{y}(t, \cdot)\|_{2}^{2} \leq C\left(T, M_{2}, L_{1}, \alpha\right) \int_{-\tau}^{t} \mathbb{E}\|\tilde{y}(s, \cdot)-\bar{y}(s, \cdot)\|_{2}^{2} d s, \forall t \in[0, T] .
$$

Therefore,

$$
\sup _{s \in[0, t]} \mathbb{E}\|\tilde{y}(s, \cdot)-\bar{y}(s, \cdot)\|_{2}^{2} \leq C\left(T, M_{2}, L_{1}, \alpha\right) \int_{-\tau}^{t} \sup _{s \in[0, t]} \mathbb{E}\|\tilde{y}(s, \cdot)-\bar{y}(s, \cdot)\|_{2}^{2} d s, \forall t \in[0, T] .
$$

By the Gronwall inequality, we obtain

$$
\sup _{s \in[0, t]} \mathbb{E}\|\tilde{y}(s, \cdot)-\bar{y}(s, \cdot)\|_{2}^{2}=0, \forall t \in[0, T] .
$$

This implies that

$$
\|\tilde{y}-\bar{y}\|_{C_{\mathscr{F}_{t}}}^{2}=\sup _{s \in[-\tau, 0]} \mathbb{E}\|\tilde{y}(s, \cdot)-\bar{y}(s, \cdot)\|_{2}^{2}+\sup _{s \in[0, T]} \mathbb{E}\|\tilde{y}(s, \cdot)-\bar{y}(s, \cdot)\|_{2}^{2}=0 .
$$

Thus, $\tilde{y}(t, x)=\bar{y}(t, x)$, a.s., for all $t \in[-\tau, T]$ and, a.e., $x \in \mathcal{O}$.

\subsection{Nonlinear Fractional Noises}

In this subsection, we prove the existence and uniqueness of mild solution to the problem (1) by the Banach Fixed Point Theorem. Different from the problem (9), the fractional noise term in (1) is a nonlinear noise. We assume that $g:[0, T] \times C_{\mathcal{F}_{t}} \rightarrow$ $\mathscr{L}_{2}^{0}\left(W, L^{2}(\mathcal{O})\right)$ satisfies 
Hypothesis 4 (H4). For any $\tilde{y}, \bar{y} \in \mathscr{D}(T)$, there exist $M_{4}, M_{5}>0$ such that

$$
\|g(t, \tilde{y}(t, \cdot))-g(t, \bar{y}(t, \cdot))\|_{\mathscr{L}_{2}^{0}}^{2} \leq M_{4} \mathbb{E}\|\tilde{y}(t, \cdot)-\bar{y}(t, \cdot)\|_{2}^{2},\|g(t, 0)\|_{\mathscr{L}_{2}^{0}} \leq M_{5},
$$

for any $t \in[0, T]$.

Theorem 2. Let $1 / 2<\alpha, \gamma, H<1$, and $N>2 \gamma$, assumptions (H1), (H2), and (H4) hold. If $\varphi \in C_{\mathscr{F}_{0}}\left([-\tau, 0] ; L^{2}(\mathcal{O})\right)$ and $v \in L^{2}([0, T] ; V)$, then there exists $T>0$ such that the problem (1) admits a unique mild solution in $\mathcal{D}(T)$.

Proof. First, we show that there exists a solution mapping. By $(\mathrm{H} 4)$, we deduce that for any $t \in[0, T]$ and $\phi \in \mathscr{D}(T)$,

$$
\begin{aligned}
\|g(t, \phi)\|_{\mathscr{L}_{2}^{0}}^{2} & =\|g(t, \phi)-g(t, 0)+g(t, 0)\|_{\mathscr{L}_{2}^{0}}^{2} \\
& \leq 2 M_{4}\|\phi\|_{C_{\mathscr{F}_{t}}}^{2}+2\|g(t, 0)\|_{\mathscr{L}_{2}^{0}} \\
& \leq C\left(M_{4}, M_{5}\right) .
\end{aligned}
$$

Thus, by Theorem 1, there exists a unique mild solution $y$ of the following problem:

$$
\left\{\begin{aligned}
& \begin{array}{l}
{ }_{0}^{C} D_{t}^{\alpha} y(t, x)+ \\
\quad
\end{array}(-\Delta)^{\gamma} y(t, x)=f\left(t, x, y_{\tau}(t, x)\right)+[\mathcal{B} v(t)](x)+g(t, \phi) \frac{d B^{H}(t)}{d t} \\
&+\int_{U} h\left(t, x, y_{\tau}(t, x) ; u\right) \tilde{\theta}(d u), t \in(0, T], x \in \mathcal{O}, \\
& y(t, x)=\varphi(t, x), t \in[-\tau, 0], x \in \mathcal{O}, \\
& y(t, x)=0, t \in[-\tau, T], x \in \mathbb{R}^{N} \backslash \mathcal{O},
\end{aligned}\right.
$$

Let

$$
\begin{aligned}
\Psi: C_{\mathscr{F}_{t}}\left([-\tau, T] ; L^{2}(\mathcal{O})\right) & \rightarrow C_{\mathscr{F}_{t}}\left([-\tau, T] ; L^{2}(\mathcal{O})\right), \\
\phi & \mapsto y .
\end{aligned}
$$

Next, we demonstrate that $\Psi$ is a contraction map. For any $\tilde{\phi}, \bar{\phi} \in \mathscr{D}(T)$, consider

$$
\|\Psi \tilde{\phi}-\Psi \bar{\phi}\|_{C_{\mathscr{F}_{t}}}^{2}=0+\sup _{t \in[0, T]} \mathbb{E}\|\Psi \tilde{\phi}(t, \cdot)-\Psi \bar{\phi}(t, \cdot)\|_{2}^{2}
$$

Further, similar to the proof of the uniqueness of mild solution to the problem (9), we get

$$
\begin{aligned}
\mathbb{E}\|\Psi \tilde{\phi}(t, \cdot)-\Psi \bar{\phi}(t, \cdot)\|_{2}^{2} & \leq 2 H t^{2 H+2 \alpha-2} \int_{0}^{t}\|g(s, \tilde{\phi})-g(s, \bar{\phi})\|_{\mathscr{L}_{2}^{0}}^{2} d s \\
& \leq 2 H M_{4} T^{2 H+2 \alpha-2}\|\tilde{\phi}-\bar{\phi}\|_{C_{\mathscr{F}_{t}}{ }^{\prime}}^{2}
\end{aligned}
$$

in review of $(\mathrm{H} 4)$. We conclude that

$$
\|\Psi \tilde{\phi}-\Psi \bar{\phi}\|_{C_{F_{t}}}^{2} \leq 2 H M_{4} T^{2 H+2 \alpha-2}\|\tilde{\phi}-\bar{\phi}\|_{C_{\mathscr{F}_{t}}}^{2} .
$$

Taking $T_{1}=\min \left\{\frac{1}{\left(4 H M_{4}\right)^{1 /(2 H+2 \alpha-2)}}, T\right\}$, then $\Psi$ is a contraction mapping on $\mathscr{D}\left(T_{1}\right)$. Furthermore, by the Banach Fixed Point Theorem, there exists a unique mild solution of problem (1) on $\left[-\tau, T_{1}\right]$. By taking $T_{1}$ as the initial time and repeating this process, we can extend the mild solution to $[-\tau, T]$ in finite steps.

\subsection{An Estimate}

According to the proof of Theorems 1 and 2, we observed that mild solutions are bounded in $\mathcal{D}(T)$. In particular, we get the following results. 
Proposition 2. Let $1 / 2<\alpha, \gamma, H<1, N>2 \gamma$, (H1), (H2) and (H3) hold. If $y$ is a mild solution of problem (9), then there exists a positive constant $M$ such that

$$
\|y\|_{C_{F_{F}}}^{2} \leq M
$$

where $M$ depends on $\alpha, T, H, M_{1}, M_{2}, M_{3},\|\varphi\|_{\mathcal{F}_{\mathcal{F}_{0}}}\|v\|_{L^{2}([0, T], V)}$ and $\|\Phi\|_{1}$.

Proof. Since $y(t, x)=\varphi(t, x)$, a.s., for all $t \in[-\tau, 0]$ and $x \in \mathcal{O}$, we obtain that

$$
\left\|\left.y\right|_{[-\tau, 0]}\right\|_{\mathcal{C}_{\mathcal{F}_{0}}}=\|\varphi\|_{C_{\mathcal{F}_{0}}} .
$$

On the other hand, for any $t \in(0, T]$, we get

$$
\begin{aligned}
\mathbb{E}\|y(t, \cdot)\|_{2}^{2} \leq & 5 \mathbb{E}\left\|\int_{\mathcal{O}} \eta(t, x-z) \varphi(0, z) d z\right\|_{2}^{2}+5 \mathbb{E}\left\|\int_{0}^{t} \int_{\mathcal{O}} \xi(t-s, x-z) \mathcal{B} v(s)(z) d z d s\right\|_{2}^{2} \\
& +5 \mathbb{E}\left\|\int_{0}^{t} \int_{\mathcal{O}} \xi(t-s, x-z) f\left(s, z, y_{\tau}(s, z)\right) d z d s\right\|_{2}^{2} \\
& +5 \mathbb{E}\left\|\int_{0}^{t} \int_{\mathcal{O}} \xi(t-s, x-z) g(s) d z d B^{H}(s)\right\|_{2}^{2} \\
& +5 \mathbb{E}\left\|\int_{0}^{t} \int_{U} \int_{\mathcal{O}} \xi(t-s, x-z) h\left(s, z, y_{\tau}(s, z) ; u\right) d z \tilde{\theta}(d u, d s)\right\|_{2}^{2} \\
& \doteq 5 \sum_{k=1}^{5} I_{k} .
\end{aligned}
$$

First, we consider

$$
\begin{aligned}
I_{1}+I_{2} & =5 \mathbb{E}\left\|\int_{\mathcal{O}} \eta(t, x-z) \varphi(0, z) d z\right\|_{2}^{2}+5 \mathbb{E}\left\|\int_{0}^{t} \int_{\mathcal{O}} \xi(t-s, x-z) \mathcal{B} v(s)(z) d z d s\right\|_{2}^{2} \\
& \leq 5\|\eta(t, \cdot)\|_{1}^{2} \mathbb{E}\|\varphi(0, \cdot)\|_{2}^{2}+5 \int_{0}^{t}\|\xi(t-s, \cdot)\|_{1}^{2} d s \int_{0}^{t}\|\mathcal{B} v(s)\|_{2}^{2} d s \\
& \leq C\|\varphi\|_{\mathcal{F}_{\mathcal{F}_{0}}}+C T^{2 \alpha-1}\|v\|_{L^{2}([0, T], V) .}
\end{aligned}
$$

Second, we get

$$
\begin{aligned}
I_{3} & \leq 5 \int_{0}^{t}\|\xi(t-s, \cdot)\|_{1}^{2} d s \mathbb{E} \int_{0}^{t}\left\|f\left(s, \cdot, y_{\tau}^{n}(s, \cdot)\right)\right\|_{2}^{2} d s \\
& \leq C T^{2 \alpha-1}\left(\|\Phi\|_{1}+M_{2}\right) \mathbb{E} \int_{-\tau}^{t}\|y(s, \cdot)\|_{2}^{2} d s .
\end{aligned}
$$

By similar discussions to (10), we obtain

$$
I_{4} \leq 4 H T^{2 H-1} \int_{0}^{t}\|\xi(t-s, \cdot)\|_{1}^{2}\|g(s)\|_{\mathscr{L}_{2}^{0}}^{2} d s \leq C M_{3} T^{2 \alpha+2 H-1} .
$$

Further,

$$
\begin{aligned}
I_{5} & \leq 5 \mathbb{E} \int_{0}^{t} \int_{U}\|\xi(t-s, \cdot)\|_{1}^{2}\left\|h\left(s, \cdot, y_{\tau}(s, \cdot) ; u\right)\right\|_{2}^{2} e(d u) d s \\
& \leq C\left(T^{2 \alpha-1}+M_{1}\right) \mathbb{E} \int_{-\tau}^{t}\|y(s, \cdot)\|_{2}^{2} d s .
\end{aligned}
$$

Therefore, we conclude that for any $t \in[-\tau, T]$,

$$
\begin{aligned}
\mathbb{E}\|y(t, \cdot)\|_{2}^{2} \leq & C\left(\alpha, T, H, M_{3},\|\varphi\|_{\left.C_{\mathcal{F}_{0}},\|v\|_{L^{2}([0, T], V)}\right)}\right. \\
& +C\left(\alpha, T, M_{1}, M_{2},\|\Phi\|_{1}\right) \mathbb{E} \int_{-\tau}^{t}\|y(s, \cdot)\|_{2}^{2} d s,
\end{aligned}
$$


where $C(\cdot)$ depends on the parameters in brackets. By taking supremum on both sides and the Granwall inequality, we get that there exists $M>0$ such that

$$
\|y\|_{\mathcal{C}_{\mathcal{F}_{t}}}^{2} \leq C\left(\alpha, T, H, M_{3},\|\varphi\|_{\mathcal{C}_{\mathcal{F}_{0}}}\|v\|_{L^{2}([0, T], V)}\right) \exp \left\{C\left(\alpha, T, M_{1}, M_{2},\|\Phi\|_{1}\right)\right\} \leq M .
$$

Proposition 3. Let $1 / 2<\alpha, \gamma, H<1$, and $N>2 \gamma$, assumptions (H1), (H2), and (H4) hold. If $y$ is a mild solution of problem (1), then there exists a positive constant $\bar{M}$ such that

$$
\|y\|_{\mathscr{C}_{\mathscr{F}_{t}}}^{2} \leq \bar{M}
$$

where $M$ depends on $\alpha, T, H, M_{1}, M_{2}, M_{4}, M_{5},\|\varphi\|_{\mathcal{C}_{\mathcal{F}_{0}}},\|v\|_{L^{2}([0, T], V)}$, and $\|\Phi\|_{1}$.

Proof. Let $\tilde{I}_{4}=\mathbb{E}\left\|\int_{0}^{t} \int_{\mathcal{O}} \xi(t-s, x-z) g\left(s, y_{\tau}(s)\right) d z d B^{H}(s)\right\|_{2}^{2}$. Then, by (H4) we get that

$$
\begin{aligned}
\tilde{I}_{4} & \leq 4 H T^{2 H-1} \int_{0}^{t}\|\xi(t-s, \cdot)\|_{1}^{2}\left\|g\left(s, y_{\tau}(s)\right)\right\|_{\mathscr{L}_{2}^{0}}^{2} d s \\
& \leq C T^{2 \alpha+2 H-1}\left\{M_{4}+M_{5} \mathbb{E} \int_{-\tau}^{t}\|y(s, \cdot)\|_{2}^{2} d s\right\} .
\end{aligned}
$$

Hence, by a similar proof to Proposition 2, we obtain

$$
\|y\|_{\mathcal{C}_{\mathcal{F}_{t}}}^{2} \leq C\left(\alpha, T, H, M_{4},\|\varphi\|_{\mathcal{C}_{\mathcal{F}_{0}}}\|v\|_{L^{2}([0, T], V)}\right) \exp \left\{C\left(\alpha, T, M_{1}, M_{2}, M_{5},\|\Phi\|_{1}\right)\right\} \leq \bar{M} .
$$

\section{Approximate Controllability}

In this section, we apply the results in Section 3 to prove the approximate controllability of problem (9) and problem (1).

Remark 2. In this section, we switch the viewpoint to clarify the presentation. By defining $[y(t)](x) \doteq y(t, x), t \in[-\tau, T]$, and $x \in \mathcal{O}$, we consider $y:[-\tau, T] \rightarrow L^{2}(\mathcal{O})$ as a mapping rather than a function. Further, define

$$
\varphi:[-\tau, 0] \rightarrow L^{2}(\mathcal{O}), f:[0, T] \times C_{\mathcal{F}_{t}} \rightarrow L^{2}(\mathcal{O}), h:[0, T] \times C_{\mathcal{F}_{t}} \times U \rightarrow L^{2}(\mathcal{O}),
$$

by $[\varphi(t)](x) \doteq \varphi(t, x),[f(t, y)](x) \doteq f(t, x, y(t, x))$, and $[h(t, y ; u)](x) \doteq h(t, x, y(t, x) ; u)$.

Let

$$
\begin{aligned}
R(T ; \varphi)= & \{y(T) \doteq y(T ; \varphi ; v): y \text { is the mild solution corresponding to } \\
& \left.v \in L^{2}([0, T] ; V) \text { and } \varphi \in C_{\mathscr{F}_{0}}\left([-\tau, 0] ; L^{2}(\mathcal{O})\right)\right\} .
\end{aligned}
$$

$R(T ; \varphi)$ denotes the set of terminate states at time $T$. For any $y(T ; \varphi ; v) \in R(T ; \varphi)$, it means that there exists a control $v$ which transfer initial state $\varphi$ to terminate state $y(T)$.

Definition 2. Problem (1) is called approximately controllable on $[0, T]$ if for any $\varphi \in C_{\mathscr{F}_{0}}\left([-\tau, 0] ; L^{2}(\mathcal{O})\right), R(T ; \varphi)$ satisfies

$$
\overline{R(T ; \varphi)}=L^{2}\left(\Sigma ; L^{2}(\mathcal{O})\right) .
$$

Let

$$
\Pi_{0}^{T} v=\int_{0}^{T} Z_{\gamma}^{\alpha}(T-s) \mathcal{B} v(s) d s,
$$




$$
\begin{aligned}
Y_{\lambda}^{T}(\cdot) & =\int_{\lambda}^{T} Z_{\gamma}^{\alpha}(T-s) \mathcal{B B}^{*}\left(Z_{\gamma}^{\alpha}\right)^{*}(T-s)(\cdot) d s, \\
G_{\lambda}^{T} & =\int_{\lambda}^{T} Z_{\gamma}^{\alpha}(T-s) \mathcal{B B}^{*}\left(Z_{\gamma}^{\alpha}\right)^{*}(T-s) d s
\end{aligned}
$$

where $\lambda \in[0, T)$. It is easy to see $\Pi_{0}^{T} \in \mathscr{L}\left(L^{2}([0, T] ; V) ; L^{2}\left(\Sigma, L^{2}(\mathcal{O})\right)\right)$. Therefore, there exists the adjoint operator denoted by $\left(\Pi_{0}^{T}\right)^{*}$. Note that $Y_{\lambda}^{T} \in \mathscr{L}\left(L^{2}\left(\Sigma, L^{2}(\mathcal{O})\right)\right.$ for any $\lambda \in[0, T)$ and for arbitrary $\psi \in L^{2}\left(\Sigma, L^{2}(\mathcal{O})\right)$,

$$
\mathrm{Y}_{0}^{T}(\psi)=\Pi_{0}^{T}\left(\Pi_{0}^{T}\right)^{*}(\psi) .
$$

$G_{\lambda}^{T}: L^{2}(\mathcal{O}) \rightarrow L^{2}(\mathcal{O})$ is called the controllability Gramian of control problem (9). Assume that

(C) For arbitrary $\lambda \in[0, T), \kappa\left(\kappa I+G_{\lambda}^{T}\right)^{-1} \in \mathscr{L}\left(L^{2}(\mathcal{O})\right)$, and

$$
\left\|\kappa\left(\kappa I+G_{\lambda}^{T}\right)^{-1}\right\| \rightarrow 0 \text { as } \kappa \rightarrow 0^{+} \text {, uniformly with respect to } \lambda \in[0, T) .
$$

Under assumption (C), the deterministic linear fully nonlocal stochastic control problem without delay corresponding to problem (9) is approximately controllable, see [42-44].

To illustrate approximate controllability of control problem (9) and problem (1), we recall the following result.

Lemma 6 ([34]). For any $\rho \in L^{2}\left(\Sigma ; L^{2}(\mathcal{O})\right)$, there exists $\zeta$ such that

$$
\rho=\mathbb{E} \rho+\int_{0}^{T} \zeta(s) d B^{H}(s),
$$

where $\zeta \in L^{2}\left(\Sigma ; L_{\mathcal{F}_{t}}^{2}\left([0, T] ; \mathscr{L}_{2}^{0}\left(W, L^{2}(\mathcal{O})\right)\right)\right)$.

Theorem 3. Let the conditions in Theorem 1 and assumption (C) hold, then stochastic control problem (9) is approximately controllable on $[-\tau, T]$.

Proof. It is necessary to prove that there exists a sequence of controls whose limit transfers initial state $\varphi$ to $y(T)$ at time $T$. The construction of the sequence is inspired by [42].

Step 1: The sequence of controls. For any positive integer $\kappa, 0<t \leq T, \rho \in$ $L^{2}\left(\Sigma ; L^{2}(\mathcal{O})\right)$ and $y \in \mathcal{D}(T)$, choose

$$
\begin{aligned}
v^{\kappa}(t ; y)= & \mathcal{B}^{*}\left(Z_{\gamma}^{\alpha}\right)^{*}(T-t)\left(\kappa I+G_{0}^{T}\right)^{-1}\left\{\mathbb{E} \rho-Y_{\gamma}^{\alpha}(T) \varphi(0)\right\} \\
& -\mathcal{B}^{*}\left(Z_{\gamma}^{\alpha}\right)^{*}(T-t) \int_{0}^{t}\left(\kappa I+G_{s}^{T}\right)^{-1} Z_{\gamma}^{\alpha}(T-s) f\left(s, y_{\tau}\right) d s \\
& -\mathcal{B}^{*}\left(Z_{\gamma}^{\alpha}\right)^{*}(T-t) \int_{0}^{t}\left(\kappa I+G_{s}^{T}\right)^{-1}\left[Z_{\gamma}^{\alpha}(T-s) g(s)-\zeta(s)\right] d B^{H}(s) \\
& -\mathcal{B}^{*}\left(Z_{\gamma}^{\alpha}\right)^{*}(T-t) \int_{0}^{t}\left(\kappa I+G_{s}^{T}\right)^{-1} Z_{\gamma}^{\alpha}(T-s) \int_{U} h\left(s, y_{\tau} ; u\right) \tilde{\theta}(d u, d s) .
\end{aligned}
$$

Let $y^{\kappa}$ be the mild solution of problem (9) associated with $v^{\kappa}$. Therefore, $y^{\kappa}(T)$ satisfies

$$
\begin{aligned}
y^{\kappa}(T)= & \rho-\kappa\left(\kappa I+G_{0}^{T}\right)^{-1}\left\{\mathbb{E} \rho-Y_{\gamma}^{\alpha}(T) \varphi(0)\right\} \\
& +\int_{0}^{T} \kappa\left(\kappa I+G_{s}^{T}\right)^{-1} Z_{\gamma}^{\alpha}(T-s) f\left(s, y_{\tau}^{\kappa}\right) d s \\
& +\int_{0}^{T} \kappa\left(\kappa I+G_{s}^{T}\right)^{-1}\left[Z_{\gamma}^{\alpha}(T-s) g(s)-\zeta(s)\right] d B^{H}(s) \\
& +\int_{0}^{T} \kappa\left(\kappa I+G_{s}^{T}\right)^{-1} Z_{\gamma}^{\alpha}(T-s) \int_{U} h\left(s, y_{\tau}^{\kappa} ; u\right) \tilde{\theta}(d u, d s) .
\end{aligned}
$$

We will show that $\left\{v^{\kappa}\right\}_{\mathcal{K}=1}^{\infty}$ is the sequence that we needed. 
Step 2: Approximate controllability. Since $\left\{y^{\kappa}\right\}_{\mathcal{K}=1}^{\infty}$ is a sequence of mild solutions, $\left\{y^{\mathcal{K}}\right\}_{\mathcal{K}=1}^{\infty}$ is bounded in $C_{\mathscr{F}_{t}}\left([-\tau, T], L^{2}(\mathcal{O})\right)$ by Proposition 2 . Therefore,

$$
\begin{aligned}
& \mathbb{E} \int_{0}^{t}\left\|f\left(s, y_{\tau}^{\kappa}(s)\right)\right\|_{2}^{2} d s+\mathbb{E} \int_{0}^{t} \int_{U}\left\|h\left(s, y_{\tau}^{\kappa}(s) ; u\right)\right\|_{2}^{2} e(d u) d s \\
\leq & 2\|\Phi\|_{1}+2 M_{2} \mathbb{E} \int_{-\tau}^{T}\left\|y^{\kappa}(s)\right\|_{2}^{2} d s \\
\leq & 2\|\Phi\|_{1}+\left(M_{1}+M_{2}\right) M(T+\tau) .
\end{aligned}
$$

Denote $f^{\kappa}(s) \doteq f\left(s, y_{\tau}^{\kappa}\right)$ and $h^{\kappa}(s ; u) \doteq h\left(s, y_{\tau}^{\kappa} ; u\right)$, then $\left\{f^{\mathcal{K}}\right\}_{\mathcal{K}=1}^{\infty}$ is bounded in $L_{\mathcal{F}_{t}}^{2}\left([0, T], L^{2}(\mathcal{O})\right)$ and $\left\{h^{\kappa}\right\}_{\mathcal{K}=1}^{\infty}$ is bounded in $M_{\mathcal{F}}^{e, 2}\left([0, T] \times U, L^{2}(\mathcal{O})\right)$. On the other hand, by assumption (C), for $\kappa>0$ sufficiently small,

$$
\left\|\kappa\left(\kappa I+G_{\lambda}^{T}\right)^{-1}\right\| \leq 1, \forall \lambda \in[0, T) .
$$

Further, we deduce that

$$
\begin{aligned}
\mathbb{E}\left\|y^{\kappa}(T)-\rho\right\|_{2}^{2} \leq & 5 \mathbb{E}\left\|\kappa\left(\kappa I+G_{0}^{T}\right)^{-1}\left\{\mathbb{E} \rho-Y_{\gamma}^{\alpha}(T) \varphi(0)\right\}\right\|_{2}^{2} \\
& +5 \mathbb{E}\left\|\int_{0}^{T} \kappa\left(\kappa I+G_{s}^{T}\right)^{-1} Z_{\gamma}^{\alpha}(T-s) f^{\kappa}(s) d s\right\|_{2}^{2} \\
& +5 \mathbb{E}\left\|\int_{0}^{T} \kappa\left(\kappa I+G_{s}^{T}\right)^{-1} Z_{\gamma}^{\alpha}(T-s) g(s) d s\right\|_{2}^{2} \\
& +5 \mathbb{E}\left\|\int_{0}^{T} \kappa\left(\kappa I+G_{s}^{T}\right)^{-1} Z_{\gamma}^{\alpha}(T-s) \int_{U} h^{\kappa}(s ; u) e(d u) d s\right\|_{2}^{2} \\
& +5 \mathbb{E}\left\|\int_{0}^{T} \kappa\left(\kappa I+G_{s}^{T}\right)^{-1} \zeta(s) d s\right\|_{2}^{2} \\
\doteq & W_{1}+W_{2}+W_{3}+W_{4}+W_{5} .
\end{aligned}
$$

By Lemma 5 and (C) we have

$$
W_{1} \leq C\left\|\kappa\left(\kappa I+G_{s}^{T}\right)^{-1}\right\|^{2}\left\{\mathbb{E}\|\rho\|_{2}^{2}+\mathbb{E}\|\varphi(0)\|_{2}^{2}\right\} \rightarrow 0 \text { as } \kappa \rightarrow 0^{+} .
$$

By the Minkowsky inequality, Lemma 5, (13), and (14), the Hölder inequality, (C), and the Lebesgue Dominated Convergence Theorem, we get

$$
\begin{aligned}
W_{2} & \leq C\left\{\mathbb{E} \int_{0}^{T}\left\|\kappa\left(\kappa I+G_{s}^{T}\right)^{-1} Z_{\gamma}^{\alpha}(T-s) f^{\kappa}(s)\right\|_{2} d s\right\}^{2} \\
& \leq C\left\{\mathbb{E} \int_{0}^{T}\left\|\kappa\left(\kappa I+G_{s}^{T}\right)^{-1}\right\|(T-s)^{s-1}\left\|f^{\kappa}(s)\right\|_{2} d s\right\}^{2} \\
& \leq C \int_{0}^{T}(T-s)^{2 \alpha-2} d s \int_{0}^{T}\left\|\kappa\left(\kappa I+G_{s}^{T}\right)^{-1}\right\|^{2} \mathbb{E}\left\|f^{\kappa}(s)\right\|_{2}^{2} d s \\
& \rightarrow 0 \text { as } \kappa \rightarrow 0^{+} .
\end{aligned}
$$

By similar discussions to $W_{2}$, we obtain

$$
W_{4} \leq C T^{2 \alpha-1} \int_{0}^{T} \int_{U}\left\|\kappa\left(\kappa I+G_{s}^{T}\right)^{-1}\right\|^{2} \mathbb{E}\left\|h^{\kappa}(s ; u)\right\|_{2}^{2} e(d u) d s \rightarrow 0 \text { as } \kappa \rightarrow 0^{+} .
$$

Further, by $(\mathrm{H} 3)$ and $\zeta \in L^{2}\left(\Sigma ; L_{\mathcal{F}_{t}}^{2}\left([0, T] ; \mathscr{L}_{2}^{0}\left(W, L^{2}(\mathcal{O})\right)\right)\right)$, we get that

$$
\begin{aligned}
W_{3}+W_{5} \leq & C H T^{2 \alpha+2 H-2} \mathbb{E} \int_{0}^{T}\left\|\kappa\left(\kappa I+G_{s}^{T}\right)^{-1}\right\|^{2}\|g(s)\|_{\mathscr{L}_{2}^{0}}^{2} d s \\
& +C H T^{2 H-1} \mathbb{E} \int_{0}^{T}\left\|\kappa\left(\kappa I+G_{s}^{T}\right)^{-1}\right\|^{2}\|\zeta(s)\|_{\mathscr{L}_{2}^{0}}^{2} d s \\
\rightarrow 0 & \text { as } \kappa \rightarrow 0^{+} .
\end{aligned}
$$


To sum up, we get

$$
\mathbb{E}\left\|y^{\kappa}(T)-\rho\right\|_{2}^{2} \rightarrow 0 \text { as } \kappa \rightarrow 0^{+} .
$$

Therefore, by the arbitrariness of $\rho \in L^{2}\left(\Sigma ; L^{2}(\mathcal{O})\right)$, we obtain the approximate controllability of problem (9) on $[-\tau, T]$.

Theorem 4. Let the conditions in Theorem 2 and (C) hold, then stochastic control problem (1) is approximately controllable on $[-\tau, T]$.

Proof. By the similar discussions to the proof of Theorem 3, we only need to show that

$$
\tilde{W}_{3} \doteq \mathbb{E}\left\|\int_{0}^{T} \kappa\left(\kappa I+G_{s}^{T}\right)^{-1} Z_{\gamma}^{\alpha}(T-s) g\left(s, y_{\tau}^{\kappa}(s)\right) d s\right\|_{2}^{2} \rightarrow 0 \text { as } \kappa \rightarrow 0 .
$$

By (12) and Proposition 3 , for any $t \in[0, T]$, we get

$$
\left\|g\left(t, y_{\tau}^{\kappa}(t)\right)\right\|_{\mathscr{L}_{2}^{0}}^{2} \leq 2 M_{4}\left\|y^{\kappa}\right\|_{\mathscr{F}_{\mathscr{F}_{t}}}^{2}+2 M_{5} \leq 2 \bar{M} M_{4}+2 M_{5}
$$

Therefore,

$$
\tilde{W}_{3} \leq C H T^{2 \alpha+2 H-2} \int_{0}^{T}\left\|\kappa\left(\kappa I+G_{s}^{T}\right)^{-1}\right\|^{2}\left\|g\left(s, y_{\tau}^{\kappa}(s)\right)\right\|_{\mathscr{L}_{2}^{0}}^{2} d s \rightarrow 0 \text { as } \kappa \rightarrow 0 .
$$

\section{Conclusions}

In this paper, we discuss a class of stochastic control problems with Caputo fractional derivatives, fractional Laplacian operators, fractional Brownian motions and Poisson jumps. We consider the problems with linear and nonlinear fractional noises by different methods. We obtain the existence and uniqueness of mild solutions when $1 / 2<\alpha, \gamma, H<1$, and $N>2 \gamma$. Furthermore, we apply the results obtained for mild solutions to get the approximate controllability of the stochastic control problems.

Author Contributions: Conceptualization, Y.F.; methodology, L.Y. and Y.F.; validation, L.Y. and Y.F.; formal analysis, L.Y.; investigation, L.Y.; writing—original draft preparation, L.Y.; writing—review and editing, Y.F.; supervision, Y.F. Both authors have read and agreed to the published version of the manuscript.

Funding: This research was funded by the National Natural Science Foundation of China Grant No. 11771107.

Acknowledgments: The authors appreciate the referees for their suggestions to this paper.

Conflicts of Interest: The authors declare no conflict of interest.

\section{References}

1. Cartea, A.; del Castillo-Negrete, D. Fluid limit of the continuous-time random walk with general Lévy jump distribution functions. Phys. Rev. E 2007, 76, 041105. [CrossRef]

2. Samko, S.G.; Kilbas, A.A.; Marichev, O.I. Fractional Integrals and Derivatives: Theory and Applications; Gordon and Breach Science Publishers: Yverdon, Switzerland, 1993.

3. Mao, X. Stochastic Differential Equations and their Applications; Horwood Publishing: Chichester, UK, 2007.

4. Paola, M.D.; Alotta, G.; Failla, G.; Pirrotta, A.; Sofi, A.; Zingales, M. The mechanically based non-local elasticity: An overview of main results and future challenges. Phil. Trans. R. Soc. A 2013, 371, 20120433. [CrossRef] [PubMed]

5. Patnaik, S.; Sidhardh, S.; Semperlotti, F. Towards a unified approach to nonlocal elasticity via fractional-order mechanics. Int. J. Mech. Sci. 2021, 189, 105992. [CrossRef]

6. Paola, M.D.; Zingales, M. Long-range cohesive interactions of non-local continuum faced by fractional calculus. Int. J. Solids Struct. 2008, 45, 5642-5659. [CrossRef]

7. Patnaik, S.; Semperlotti, F. A generalized fractional-order elastodynamic theory for non-local attenuating media. Proc. A 2020, 476, 20200200. [CrossRef] [PubMed] 
8. Patnaik, S.; Hollkamp, J.P.; Semperlotti, F. Applications of variable-order fractional operators: a review. Proc. R. Soc. A 2020, 476, 20190498. [CrossRef] [PubMed]

9. Sun, H.; Chang, A.; Zhang, Y.; Chen, W. A review on variable-order fractional differential equations: mathematical foundations, physical models, numerical methods and applications. Fract. Calc. Appl. Anal. 2019, 22, 27-59. [CrossRef]

10. Ding, W.; Patnaik, S.; Sidhardh, S.; Semperlotti, F. Applications of distributed-order fractional operators: A Review. Entropy 2021, 23, 110. [CrossRef]

11. Liang, Y.; Chen, W.; Xu, W.; Sun, H. Distributed order Hausdorff derivative diffusion model to characterize non-Fickian diffusion in porous media. Commun. Nonlinear Sci. Numer. Simul. 2019, 70, 384-393. [CrossRef] 
12. Wang, B. Dynamics of fractional stochastic reaction-diffusion equations on unbounded domains driven by nonlinear noise. J. Differ. Equs. 2019, 268, 1-59. [CrossRef]

13. Mohammed, W.W. Approximate solutions for stochastic time-fractional reaction-diffusion equations with multiplicative noise. Math. Meth. Appl. Sci. 2021, 44, 2140-2157. [CrossRef]

14. Reutskiy, S.; Zhang, Y.; Lin, J.; Sun, H. Novel numerical method based on cubic B-splines for a class of nonlinear generalized telegraph equations in irregular domains. Alex. Eng. J. 2020, 59, 77-90. [CrossRef]

15. Reutskiy, S.; Zhang, Y.; Lin, J.; Sun, H. A Ritz-based finite element method for a fractional-order boundary value problem of nonlocal elasticity. Int. J. Solids Struct. 2020, 202, 398-417.

16. Li, C.; Zeng, F. Numerical Methods for Fractional Calculus; CRC Press: Boca Raton, FL, USA, 2015.

17. Alotta, G.; Failla, G.; Zingales, M. Finite-element formulation of a nonlocal hereditary fractional-order Timoshenko beam. J. Eng. Math. 2017, 143, D4015001. [CrossRef]

18. Alotta, G.; Failla, G.; Zingales, M. Finite element method for a nonlocal Timoshenko beam model. Finite Elem. Anal. Des. 2014, 89, 77-92. [CrossRef]

19. Kemppainen, J.; Siljander, J.; Zacher, R. Representation of solutions and large-time behavior for fully nonlocal diffusion equations. J. Differ. Equs. 2017, 263, 149-201. [CrossRef]

20. Li, L.; Liu, J.G.; Wang, L. Cauchy problems for Keller-Segel type time-space fractional diffusion equation. J. Differ. Equs. 2018, 265, 1044-1096. [CrossRef]

21. Pozo, J.; Vergara, V.C. Fundamental solutions and decay of fully non-local problems. Discret. Contin. Dyn. Syst. 2019, 39, 639-666. [CrossRef]

22. Lakhel, E.H.; Hajji, S. Existence and uniqueness of mild solutions to neutral SFDEs driven by a fractional Brownian motion with non-Lipschitz coefficients. J. Numer. Math. Stoch. 2015, 7, 14-29.

23. Li, K.X. Stochastic delay fractional evolution equations driven by fractional Brownian motion. Math. Methods Appl. Sci. 2014, 38, 1582-1591. [CrossRef]

24. Pei, B.; Xu, Y. Mild solutions of local non-Lipschitz stochastic evolution equations with jumps. Appl. Math. Lett. 2016, 52, 80-86. [CrossRef]

25. Caraballo, T.; Garrido-Atienza, M.; Taniguchi, T. The existence and exponential behavior of solutions to stochastic delay evolution equations with a fractional Brownian motion. Nonlinear Anal. 2011, 74, 3671-3684. [CrossRef]

26. Gao, H.; Garrido-Atienza, M.J.; Schmalfuss, B. Random attractors for stochastic evolution equations driven by fractional Brownian motion. SIAM J. Math. Anal. 2014, 46, 2281-2309. [CrossRef]

27. Hesse, R.; Neamţu, A. Local mild solutions for rough stochastic partial differential equations. J. Differ. Equs. 2019, 267, 6480-6538. [CrossRef]

28. Babiarz, A.; Niezabitowski, M. Controllability problem of fractional neutral systems: A survey. Math. Probl. Eng. 2017, 2017, 4715861. [CrossRef]

29. Zhang, R.; Guo, L. Controllability of stochastic game-based control systems. SIAM J. Control Optim. 2019, 57, 3799-3826. [CrossRef]

30. Lakhel, E.H. Controllability of neutral stochastic functional integro-differential equations driven by fractional Brownian motion. Stoch. Anal. Appl. 2016, 34, 427-440. [CrossRef]

31. Ahmed, H. Approximate controllability via resolvent operators of Sobolev-type fractional stochastic integrodifferential equations with fractional Brownian motion and Poisson jumps. Bull. Iran. Math. Soc. 2019, 45, 1045-1059. [CrossRef]

32. Shu, L.; Shu, X.B.; Mao, J. Approximate controllability and existence of mild solutions for Riemann-Liouville fractional stochastic evolution equations with nonlocal conditions of order $1<\alpha<2$. Fract. Calc. Appl. Anal. 2019, 22, 1086-1112.

33. Shukla, A.; Sukavanam, N.; Pandey, D.N. Approximate controllability of semilinear fractional stochastic control system. Asian Eur. J. Math. 2018, 11, 1-15. [CrossRef]

34. Dauer, J.; Mahmudov, N. Controllability of stochastic semilinear functional differential equations in Hilbert spaces. J. Math. Anal. Appl. 2004, 290, 373-394. [CrossRef]

35. Sakthivel, R.; Ganesh, R.; Ren, Y.; Anthoni, S. Approximate controllability of nonlinear fractional dynamical systems. Commun. Nonlinear Sci. Numer. Simul. 2013, 18, 3498-3508. [CrossRef]

36. Sakthivel, R.; Suganya, S.; Anthoni, S.M. Approximate controllability of fractional stochastic evolution equations. Comput. Math. Appl. 2012, 63, 660-668. [CrossRef]

37. Nualart, D. Stochastic calculus with respect to fractional Brownian motion. Ann. Fac. Sci. Toulouse Math. 2006, 15, 63-78. [CrossRef]

38. Boufoussi, B.; Hajji, S. Neutral stochastic functional differential equations driven by a fractional Brownian motion in a hilbert space. Stat. Probab. Lett. 2012, 82, 1549-1558. [CrossRef]

39. Chow, P. Stochastic Partial Differential Equations, 2nd ed.; Advances in Applied Mathematics; CRC Press: Boca Raton, FL, USA, 2015.

40. Nezza, E.; Palatucci, G.; Valdinoci, E. Hitchhiker's guide to the fractional sobolev spaces. Bull. Sci. Math. 2012, 136, 521-573. [CrossRef] 
41. Agarwal, R.P.; dos Santos, J. P. C.; Cuevas, C. Analytic resolvent operator and existence results for fractional integro-differential equations. J. Abstr. Differ. Equ. Appl. 2012, 2, 26-47.

42. Mahmudov, N.I. Controllability of linear stochastic systems in Hilbert spaces. J. Math. Anal. Appl. 2001, 259, 64-82. [CrossRef]

43. Mahmudov, N.I.; Zorlu, S. Controllability of non-linear stochastic systems. Int. J. Control 2003, 76, 95-104. [CrossRef]

44. Zabczyk, J. Mathematical Control Theory: Deterministic Finite Dimensional Systems; Springer: New York, NY, USA, 1990. 